\title{
Morphological features of parapapillary beta zone and gamma zone in chronic primary angle-closure glaucoma
}

\author{
Kunte Shang ${ }^{1,2} \cdot$ Xinxin $\mathrm{Hu}^{1,2} \cdot \mathrm{Yi} \mathrm{Dai}^{1,2}$
}

Received: 7 February 2019 / Revised: 11 April 2019 / Accepted: 27 April 2019 / Published online: 30 August 2019

(C) The Author(s), under exclusive licence to The Royal College of Ophthalmologists 2019

\section{Learning Objectives}

Upon completion of this activity, participants will be able to:

1. Describe morphological features of the parapapillary beta and gamma zone in eyes with CACG compared with nonmyopic control eyes, according to an observational cross-sectional study

2. Determine factors associated with morphological features of the parapapillary beta and gamma zone, according to an observational cross-sectional study

3. Identify pathophysiological implications of morphological features of the parapapillary beta and gamma zone in eyes with CACG, according to an observational cross-sectional study

\section{Accreditation Statements}

In support of improving patient care, this activity has been planned and implemented by Medscape, LLC and Springer Nature. Medscape, LLC is jointly accredited by the Accreditation Council for Continuing Medical Education (ACCME), the Accreditation Council for Pharmacy Education (ACPE), and the American Nurses Credentialing Center (ANCC), to provide continuing education for the healthcare team.

Medscape, LLC designates this Journal-based CME activity for a maximum of 1.0 AMA PRA Category 1 Credit(s) ${ }^{\mathrm{TM}}$. Physicians should claim only the credit commensurate with the extent of their participation in the activity.

\section{Credit Hours}

1.0

Release date: 30 August 2019

Expiration date: 30 August 2020

Post-test link: https://medscape.org/eye/posttest 915887

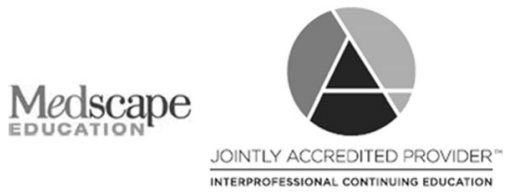

Yi Dai

ydai@fudan.edu.cn

1 Department of Ophthalmology \& Visual Science, Eye \& ENT Hospital, Shanghai Medical College, Fudan University, Shanghai 200031, China
2 NHC Key Laboratory of Myopia (Fudan University), Key Laboratory of Myopia, Chinese Academy of Medical Sciences, and Shanghai Key Laboratory of Visual Impairment and Restoration (Fudan University), Shanghai 200031, China 


\section{Authors/Editor disclosure information}

Sobha Sivaprasad has disclosed the following relevant financial relationships: Served as an advisor or consultant for: Allergan, Inc.; Bayer AG; Boehringer Ingelheim Pharmaceuticals, Inc.; Heidelberg Pharma GmbH; Optos; Roche. Served as a speaker or a member of a speakers bureau for: Allergan, Inc.; Bayer AG; Novartis Pharmaceuticals Corporation; Optos. Received grants for clinical research from: Allergan, Inc.; Bayer AG; Boehringer Ingelheim Pharmaceuticals, Inc.; Novartis Pharmaceuticals Corporation; Optos. Kunte Shang, MD, has disclosed no relevant financial relationships. Xinxin Hu, MD, has disclosed no relevant financial relationships. Yi Dai, $\mathrm{MD}, \mathrm{PhD}$, has disclosed no relevant financial relationships.

\section{Journal CME author disclosure information}

Laurie Barclay, MD, has disclosed no relevant financial relationships.

\section{Abstract}

Purpose To investigate the morphological features of parapapillary beta zone and gamma zone and their associated factors in eyes with chronic primary angle-closure glaucoma (CACG).

Methods The observational cross-sectional study included 65 CACG eyes and 65 non-myopic control eyes. On enhanced depth imaging of optical coherent tomography images, the area of parapapillary beta zone and gamma zone, and the peripapillary choroidal thickness at 6 sectors were measured. The optic disc ovality index and Bruch's membrane opening (BMO) shape were further calculated.

Results Beta zone was present in 103 (79.2\%) eyes and gamma zone in 29 (22.3\%) eyes. Compared to control eyes, CACG was associated with larger parapapillary beta zone, female gender, and older age $(P<0.01)$. No significant difference was observed in axial length and peripapillary choroidal thickness between both groups $(P>0.05)$. In multivariate analysis, beta zone area was positively associated with older age and higher prevalence of CACG $(P<0.01)$, while a larger gamma zone area was associated with a smaller disc ovality index and a higher BMO ovality ratio $(P<0.01)$. The peripapillary choroidal thickness at six sectors was decreased with older age $(P<0.01)$.

Conclusions In mainly non-myopic subjects with or without CACG, larger parapapillary beta zone was correlated with older age and presence of glaucoma, while a larger parapapillary gamma zone was correlated with disc ovality but not with glaucoma. Parapapillary beta zone and gamma zone may play different roles in physiological and glaucomatous changes around optic nerve head.

\section{Introduction}

The conventional ophthalmoscopic beta zone [1] of parapapillary atrophy characterized by visible choroidal vessels and the sclera has recently been divided into two subzones: gamma zone and (new) beta zone [2,3]. Based on spectral domain optical coherence tomography (SD-OCT) findings, gamma zone could be distinguished as the parapapillary region free of Bruch's membrane, and beta zone could be distinguished by the presence of Bruch's membrane and absence of RPE. Both gamma zone and beta zone were whitish zones upon ophthalmoscopy, while a delicate color difference could be noticed to distinguish these two subzones occasionally.

Growing evidence has suggested that parapapillary beta zone was associated mainly with primary open angle glaucoma and to a lower degree with axial myopia, while parapapillary gamma zone was strongly correlated with axial myopia [3-5]. Studies on the characteristics of SDOCT defined parapapillary atrophy in eyes with primary angle-closure glaucoma have only scarcely been performed [6], if at all. Moreover, the etiology of the parapapillary beta and gamma zone in glaucoma has remained elusive so far.
Using the enhanced depth imaging of SD-OCT technology, we therefore performed this study to investigate the morphological features of parapapillary beta zone and gamma zone and associated factors in eyes with chronic primary angle-closure glaucoma (CACG).

\section{Methods}

\section{Subjects}

The study population consisted of patients with CACG and normal individuals attending or visiting the Shanghai Eye, Ear, Nose and Throat Hospital of the Fudan University. The study protocol and data collection adhered to the tenets of the Declaration of Helsinki and was approved by the Institutional Review Board. All study participants consented to the ophthalmologic examinations before they were performed.

CACG was diagnosed according to the International Society for Geographical and Epidemiological Ophthalmology (ISGEO) system [7]. The diagnostic criteria were an increased intraocular pressure, a synechial closure of the 
anterior chamber angle upon gonioscopy, no history and no ocular signs of an acute glaucoma attack, and glaucomatous optic nerve head changes with corresponding visual field defects. Exclusion criteria were the diagnosis of secondary angle-closure glaucoma, a combined pathomechanism for glaucomatous optic neuropathy, and any ophthalmic diseases other than glaucoma (e.g., diabetic retinopathy). For those patients with bilateral CACG, only one eye randomly selected was included into the study.

All individuals underwent a comprehensive ophthalmologic examination including the assessment of visual acuity, refractometry, slit-lamp biomicroscopy, Goldmann applanation tonometry, biometry of ocular parameters including axial length, central cornea thickness and anterior chamber depth (Lenstar LS 900; Haag-Streit Diagnostics, USA), color photography of the optic nerve head (CR-DGI; Canon, Inc., Tokyo, Japan), and OCT-scanning of the fovea and optic disc and measurement of the peripapillary retinal nerve fiber layer thickness using spectral-domain optical coherence tomography (SD-OCT) (Spectralis OCT, Heidelberg Engineering, Heidelberg, Germany). Gonioscopy (Goldmann-type one-mirror lens, Haag Streit, Switzerland) and standard automatic perimetry (Carl Zeiss-Humphrey Systems, Dublinand, USA) were performed for all CACG patients.

\section{Determination of parapapillary beta zone and gamma zone}

We used the Spectralis HRA + OCT system to scan the optic disc region. The images were captured in a rectangle of $15^{\circ} \times 15^{\circ}$ for horizontal scans including the whole optic disc and peripapillary atrophy region. This rectangle was scanned with $37 \mathrm{~B}$-scans, the distance between each section was $121 \mu \mathrm{m}$. We also captured 24 radial lines B-scans with a $7.5^{\circ} \mathrm{B}$-scan angle to cover the whole optic disc region and the peripapillary region by the enhanced depth imaging mode. The ART mode of both rectangle and radial scans were set to 24 images averaged with a quality score more than 15. Moreover, we captured a single scan of 100 ART mode if necessary.

Within the parapapillary region, one has differentiated between alpha, beta and gamma zones, as previously described [3]. The alpha zone was characterized by the presence of Bruch's membrane covered by an irregular RPE, in beta zone Bruch's membrane was void of RPE cells, and gamma zone extended between the end of Bruch's membrane and the inner border of peripapillary ring as the optic disc border. We assessed the area of beta zone and gamma zone using the measurement tools built into the software of the Spectralis HRA + OCT. To avoid an error of measurement, the determination of the Bruch's membrane opening (BMO), the delineation of the various zones and the disc margin was performed by two experienced examiners masked to each other (Figs. 1, 2).

On the B-scan OCT images, we measured the minimal and maximal disc diameters, as well as the minimal and maximal BMO diameters, using the built-in measurement tools. The ratio of the minimal-to-maximal disc diameter was calculated as the optic disc ovality index. The ratio of the minimal-to-maximal BMO diameter was calculated as the BMO ovality ratio. As previously described [8], the optic disc diameter was defined as the length between the two borders marked on Bruch's membrane in eyes without a parapapillary gamma zone, that is, in eyes in which the peripapillary ring was covered by Bruch's membrane. In eyes with a gamma zone (i.e., eyes in which Bruch's membrane did not extend to the border of the peripapillary ring), the peripapillary ring was defined to be the optic disc border.

\section{Measurements of peripapillary choroidal thickness (PCT) and circumpapillary retinal nerve fiber layer thickness (cpRNFL)}

The PCT was measured in 6 meridians corresponding to the nasal-superior, nasal, nasal-inferior, temporal-superior, temporal, and temporal-inferior sectors. In each meridian, the cross-sectional area of the choroid within a distance of $500 \mu \mathrm{m}$ from the peripapillary border tissue of Elschnig was measured using the built-in drawing tool of the Spectralis viewer software. The sector PCT was obtained by dividing the measured choroidal area by $500 \mu \mathrm{m}$. The mean PCT was calculated as the average of the 6 sector PCTs. Measurements were performed twice by each of the two masked observers, and the mean of the measurements was taken for statistical analysis. The values of the cpRNFL in the corresponding sectors were generated by the in-built software of Spectralis OCT automatically.

\section{Statistical analysis}

All statistical analyses were performed using SPSS software 25.0 (IBM-SPSS, Inc., Chicago, IL, USA). The means, standard deviations and ranges of the main outcome parameters were presented. First, a one-way analysis of variance was used to evaluate parameters between CACG and control groups. Second, we performed a univariate logistic regression to analyze parameters that influence the presence of beta zone and gamma zone. Third, we performed a univariate linear regression analysis to assess associations between the area of gamma and beta zone, PCT and the main systemic and ocular parameters. Then we conducted a multivariate linear regression analysis with those parameters which showed significant associations in the univariate analysis as independent parameters. We dropped step by 

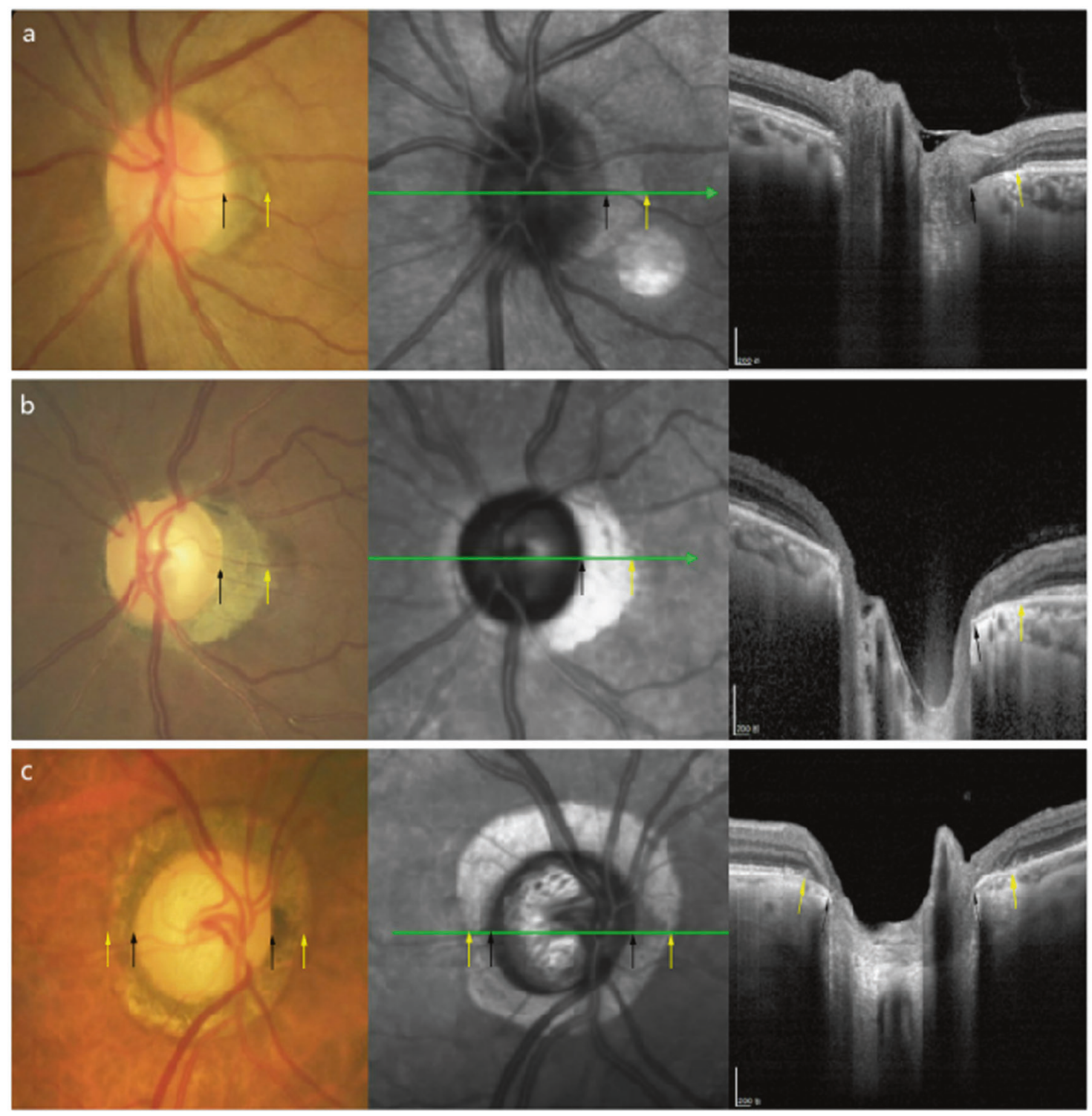

Fig. 1 Fundus photograph and optical coherence tomography images of the parapapillary beta zone (between black and yellow arrows) in the normal and CACG eyes. Parapapillary beta zone often locates in

step those independent parameters that no longer showed significant associations. All $P$-values were two-sided and the statistical significance level was set at 0.05 .

\section{Results}

The study included 65 normal eyes of 65 subjects and 65 eyes of 65 patients with CACG. The mean age was $57.04 \pm$ 12.47 years (median, 58 years; range, 22-80 years), and the mean axial length was $22.69 \pm 0.83 \mathrm{~mm}$ (median, $22.67 \mathrm{~mm}$; range, $20.45-24.41 \mathrm{~mm}$ ). All eyes were phakic.

Compared with the control group, the mean age of the CACG group (59.91 \pm 10.45 years; range, $35-80$ years) was temporal region, and some are circular shape in chronic primary angleclosure eyes

significantly older $(P=0.01)$ than that of the control group (54.21 \pm 13.67 years; range, $22-77$ years). The beta zone area in the CACG group $\left(0.97 \pm 0.93 \mathrm{~mm}^{2}\right.$; range, $\left.0-3.59 \mathrm{~mm}^{2}\right)$ was significantly larger $(P<0.001)$ than that in the control group $\left(0.41 \pm 0.53 \mathrm{~mm}^{2}\right.$; range, $\left.0-2.65 \mathrm{~mm}^{2}\right)$. Both groups did not vary significantly in axial length $(P=$ $0.64)$, refractive error $(P=0.63)$ and mean PCT $(P=0.14)$ (Table 1, Fig. 3).

In the whole study cohort, 103 eyes had a beta zone (mean area $0.69 \pm 0.81 \mathrm{~mm}^{2}$, range $0.10-4.84 \mathrm{~mm}^{2}$ ), including $60(92.3 \%)$ eyes in the CACG group and 43 $(66.1 \%)$ eyes in the control group. With regard to the beta zone locations, $13(12.0 \%)$ eyes present with a circular beta zone and $90(87.4 \%)$ eyes with beta zone located only at the 
temporal optic disc border. In univariate logistic regression analysis, the presence of beta zone was associated with age $(P=0.021)$, presence of CACG $(P<0.001)$, mean cpRNFL thickness $(P<0.001)$ and central cornea thickness $(P=0.042)$. The presence of gamma zone was associated with disc ovality index $(P<0.001)$ and BMO ovality ratio $(P=0.001)$.

To investigate the factors associated with the size of beta zone, we performed the univariate and multivariate linear regression analysis on beta zone area. In univariate analysis, a larger beta zone area was significantly associated with older age $(P<0.001)$, higher prevalence of CACG $(P<$ $0.001)$, thinner mean PCT $(P=0.002)$, thinner cpRNFL thickness $(P<0.001)$, thinner central cornea thickness $(P=$ $0.003)$, and thicker lens thickness $(P=0.047)$. Model building for multivariate analysis began with the list of independent parameters including age, presence of CACG,

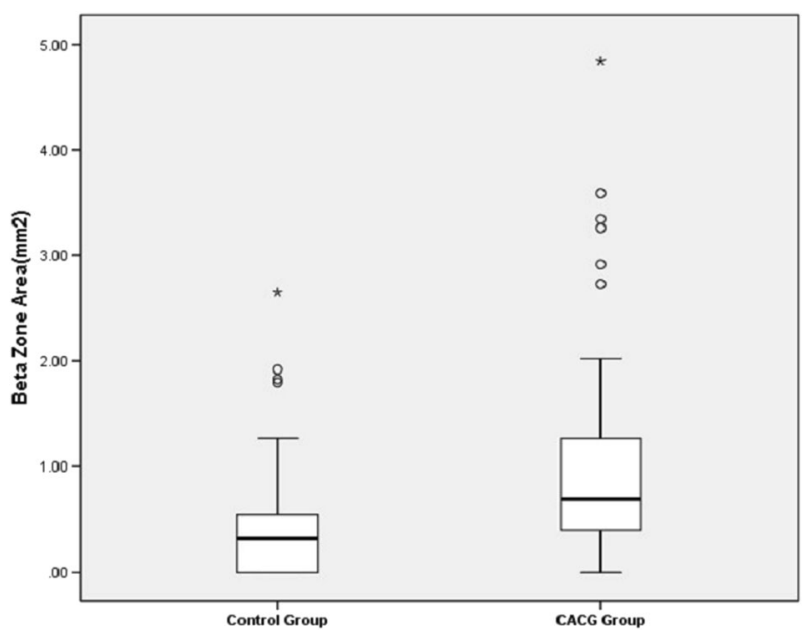

Fig. 2 Boxplots showing the distribution of the parapapillary beta zone area in the control group and in the chronic primary angle-closure glaucoma (CACG) group

Table 1 Main ocular parameters between chronic primary angleclosure glaucoma (CACG) and control eyes mean PCT, cpRNFL thickness, central cornea thickness and lens thickness. From this full model, non-significant parameters were removed step by step beginning with the parameter with the highest $P$-value (cpRNFL thickness $P=$ 0.72 ). With the reduced list of independent parameters, the multivariate analysis was repeated, leading to the exclusion of mean PCT $(P=0.13)$ and lens thickness $(P=0.24)$, until eventually all remaining independent parameters showed a significant association with beta zone area. In final model $\left(R^{2}=0.257\right)$, a larger beta zone area was associated with older age $(P=0.001)$, higher prevalence of CACG $(P=$ $0.016)$ and thinner central corneal thickness $(P=0.03)$ (Table 2).

In the whole study cohort, 29 eyes had a gamma zone (mean area $0.37 \pm 0.20 \mathrm{~mm}^{2}$, range $0.11-0.73 \mathrm{~mm}^{2}$ ), including $13(20.0 \%)$ eyes in the CACG group and 16 (24.6\%) eyes in the control group. With regard to the gamma zone locations, $12(41.4 \%)$ eyes present with a nasal gamma zone, $6(20.7 \%)$ eyes present with a temporal gamma zone, and 11 (37.9\%) eyes present with an inferior gamma zone. In univariate linear regression analysis, larger gamma zone area was associated with a smaller disc ovality index $(P<0.001)$ and higher BMO ovality ratio $(P=0.001)$. In the multivariate linear regression analysis $\left(R^{2}=0.680\right)$, a larger gamma zone area was associated with a smaller disc ovality index $(P<0.001)$ and higher BMO ovality ratio $(P<0.001)$ (Fig. 4$)$.

To further investigate the relationship between PCT and parapapillary beta zone, we performed the univariate and multivariate linear regression analysis on PCT in eyes with beta zone. In univariate analysis, thicker PCT in the temporal sector and nasal sector were only associated with younger age. The PCT in the temporal inferior, temporal superior, nasal inferior, and nasal superior sectors were associated with age and beta zone area. In further multivariate analysis, the parameter of presence of CACG was

\begin{tabular}{lccr}
\hline Parameters & CACG group $(n=65)$ & Control group $(n=65)$ & $P$-value \\
\hline Age $($ year $)$ & $59.91 \pm 10.45$ & $54.21 \pm 13.67$ & 0.008 \\
Axial length $(\mathrm{mm})$ & $22.65 \pm 0.87$ & $22.72 \pm 0.80$ & 0.636 \\
Spherical equivalent(D) & $0.088 \pm 0.425$ & $0.049 \pm 0.437$ & 0.691 \\
cpRNFL thickness $(\mu \mathrm{m})$ & $60.79 \pm 13.99$ & $118.53 \pm 15.47$ & $<0.001$ \\
Beta zone area $\left(\mathrm{mm}^{2}\right)$ & $0.97 \pm 0.93$ & $0.41 \pm 0.53$ & $<0.001$ \\
Gamma zone area $\left(\mathrm{mm}^{2}\right)$ & $0.073 \pm 0.176$ & $0.093 \pm 0.183$ & 0.517 \\
Mean PCT $(\mu \mathrm{m})$ & $114.61 \pm 42.24$ & $124.33 \pm 28.59$ & 0.137 \\
PCT nasal superior $(\mu \mathrm{m})$ & $114.02 \pm 43.84$ & $126.62 \pm 32.67$ & 0.064 \\
PCT nasal $(\mu \mathrm{m})$ & $113.24 \pm 40.56$ & $121.08 \pm 41.88$ & 0.279 \\
PCT nasal inferior $(\mu \mathrm{m})$ & $104.52 \pm 49.00$ & $116.32 \pm 30.25$ & 0.099 \\
PCT temporal superior $(\mu \mathrm{m})$ & $119.79 \pm 47.33$ & $134.52 \pm 39.20$ & 0.054 \\
PCT temporal $(\mu \mathrm{m})$ & $119.26 \pm 48.41$ & $116.56 \pm 40.19$ & 0.729 \\
PCT temporal inferior $(\mu \mathrm{m})$ & $106.12 \pm 49.79$ & $118.76 \pm 31.49$ & 0.085 \\
\hline
\end{tabular}



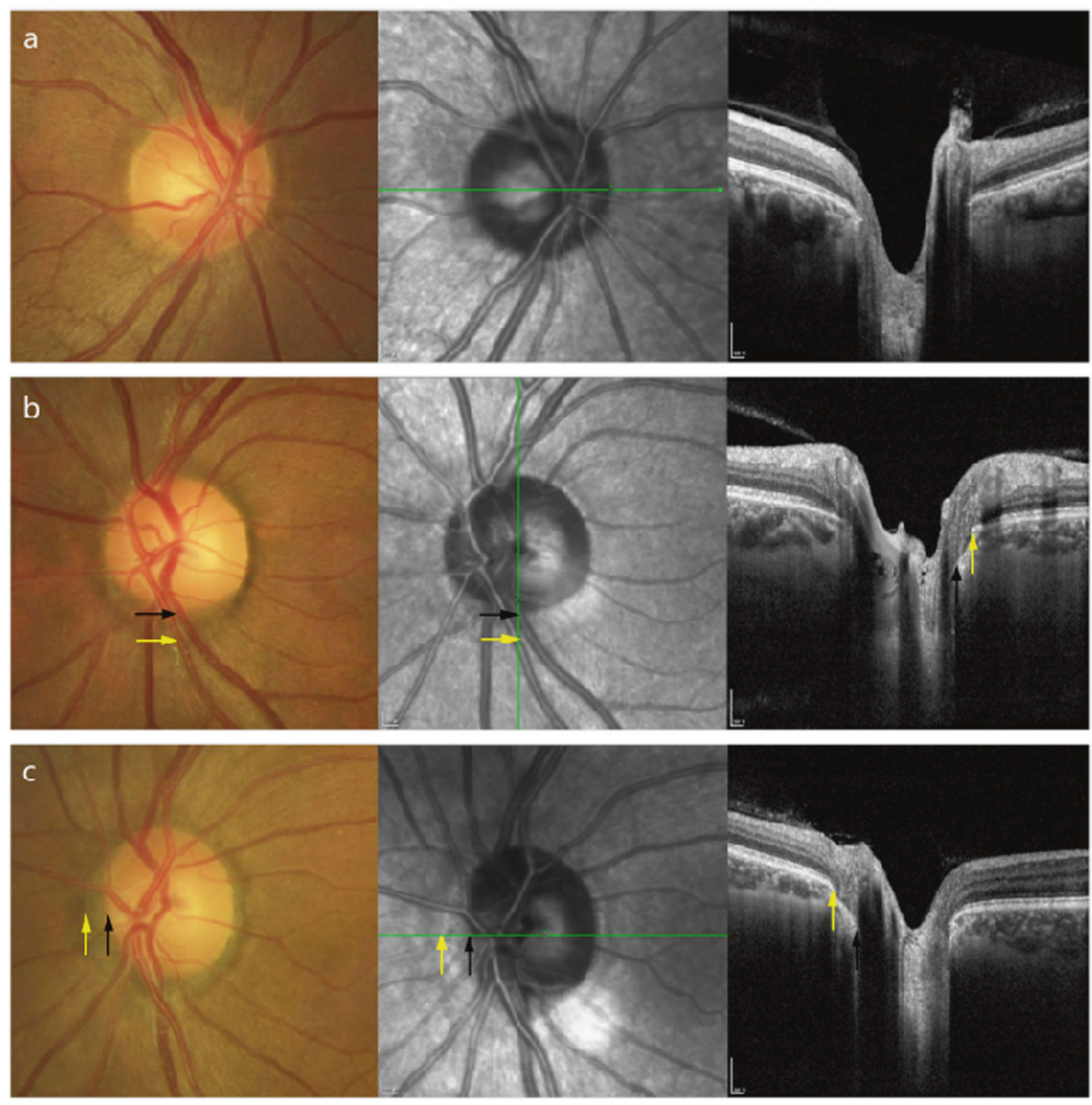

Fig. 3 Fundus photograph and optical coherence tomography images of the parapapillary gamma zone (between black and yellow arrows) in the non-myopic eyes. A majority of non-myopic eyes does not develop

added. From full model, we then removed non-significant terms step by step beginning with the parameter with the highest $P$-value. The presence of CACG and beta zone area were removed step by step. In the final model, thinner PCT at temporal superior sectors were related with older age and larger beta zone area. Thinner PCT at temporal inferior, nasal inferior, and nasal superior sectors was only related to older age (Table 3).

\section{Discussion}

Our findings demonstrated that in mainly non-myopic eyes with or without CACG, a larger parapapillary beta zone was associated with older age and presence of glaucoma, while a gamma zone (a). While the presence of gamma zone, which is composed by externally oblique border tissue, located quite often in peripapillary inferior (b) and nasal (c) regions in non-myopic eyes

larger parapapillary gamma zone was associated with a smaller disc ovality index. The PCT was negatively associated with age, but not associated with the presence of CACG or peripapillary RNFL thickness.

Age-related changes in parapapillary beta zone and the peripapillary choroid thickness were observed in our study. However, the CACG-related changes were observed for beta zone, but nor for the PCT. Previous studies have suggested a close relationship between peripapillary choroidal thickness and beta zone in POAG [9-11]. SullivanMee et al. [9] reported that the juxtapapillary choroidal volume was decreased in POAG eyes with beta zone in comparison with normal eyes with beta zone, suggesting beta zone might be a biomarker for a peripapillary deep vascular compromise in POAG. Lee et al. [10] reported that 
Table 2 Linear regression analysis of parameters associated with peripapillary beta zone area and gamma zone area

\begin{tabular}{|c|c|c|c|c|}
\hline \multirow[t]{2}{*}{ Parameter } & \multicolumn{2}{|c|}{ Univariate analysis } & \multicolumn{2}{|c|}{ Multivariate analysis } \\
\hline & $P$-value & Standardized coefficient beta & $P$-value & Standardized coefficient beta \\
\hline \multicolumn{5}{|l|}{ Beta zone area } \\
\hline Age (year) & $<0.001$ & 0.372 & 0.001 & 0.300 \\
\hline Axial length (mm) & 0.182 & 0.119 & & \\
\hline CACG & $<0.001$ & 0.349 & 0.016 & 0.228 \\
\hline Optic disc ovality index & 0.858 & 0.016 & & \\
\hline BMO ovality ratio & 0.108 & -0.144 & & \\
\hline Mean PCT $(\mu \mathrm{m})$ & 0.002 & -0.280 & & \\
\hline cpRNFL thickness & $<0.001$ & -0.347 & & \\
\hline Central cornea thickness $(\mu \mathrm{m})$ & 0.003 & -0.290 & 0.030 & -0.199 \\
\hline Lens thickness (mm) & 0.047 & 0.228 & & \\
\hline \multicolumn{5}{|l|}{ Gamma zone area } \\
\hline Age (year) & 0.134 & -0.132 & & \\
\hline Axial length (mm) & 0.659 & -0.040 & & \\
\hline CACG & 0.517 & -0.057 & & \\
\hline Optic disc ovality index & $<0.001$ & -0.404 & $<0.001$ & -0.926 \\
\hline BMO ovality ratio & 0.001 & 0.287 & $<0.001$ & 0.803 \\
\hline Mean PCT $(\mu \mathrm{m})$ & 0.501 & 0.061 & & \\
\hline cpRNFL thickness & 0.505 & 0.059 & & \\
\hline Central cornea thickness $(\mu \mathrm{m})$ & 0.116 & 0.157 & & \\
\hline Lens thickness (mm) & 0.750 & 0.037 & & \\
\hline
\end{tabular}

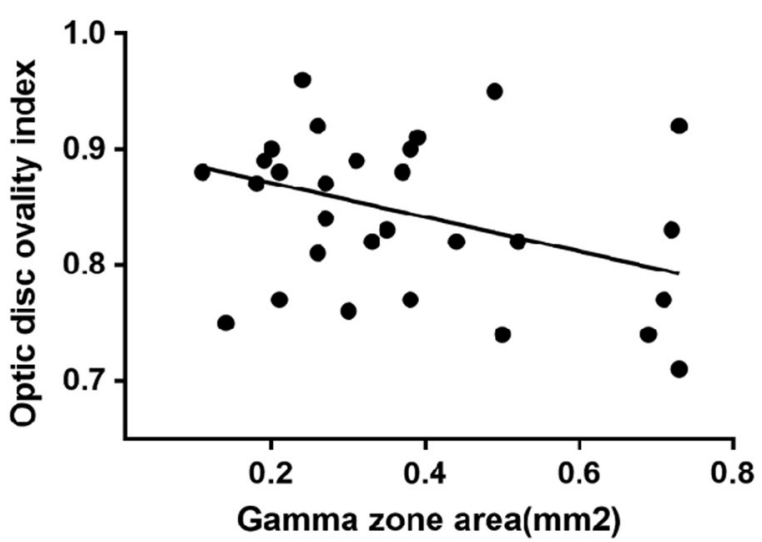

Fig. 4 Scatterplot showing the correlation between parapapillary gamma zone area and optic disc ovality index $\left(R^{2}=0.157\right)$

PCT in the POAG group was significantly thinner than that in a healthy control group in the inferotemporal location, which was coincident with the location of the larger beta zone. In our study, however, such differences on PCT were not observed between CACG and normal eyes, even though beta zone parapapillary atrophy was more prevalent and larger in CACG vs. normal eyes. A possible explanation is that although both are considered to be a chronic pathologic process, the patterns and processes of glaucomatous damage in CACG are different from those in POAG. The typical development of CACG is angle-closure, intraocular pressure elevation and optic nerve damage. In contrast, in many eyes with POAG (including eyes with normal-tension glaucoma), vascular factors have been considered to be factors leading to the optic neuropathy [12-14]. Considering that the peripapillary choroid is nourished by branches of the short posterior ciliary arteries which also supply the deep optic nerve head structures via the arterial circle of Zinn-Haller [15], a peripapillary choroidal atrophy might be linked to microcirculatory defects around the optic nerve head. Taken together, a peripapillary choroidal vascular defect may not be a causal factor in the formation of beta zone in eyes with CACG.

The conventional ophthalmoscopic beta zone has long been considered to be related with age and glaucoma [16-18]. Recent studies on SD-OCT defined beta and gamma zone further suggested that the (new) beta zone is related with POAG, while gamma zone is mainly related with myopia. Our previous study [3] showed that beta zone was associated with the presence of POAG and longer axial length in mainly myopic eyes with or without POAG. The average area of beta zone was $0.85 \pm 0.60 \mathrm{~mm}^{2}$ in POAG eyes with an average axial length of $25.6 \pm 2.4 \mathrm{~mm}$. Current data showed that beta zone was associated with the presence of CACG and older age in mainly non-myopic eyes. The average area of beta zone was $0.93 \pm 0.73 \mathrm{~mm}^{2}$ in CACG eyes with an average axial length of $22.66 \pm 0.82 \mathrm{~mm}$. The average area of beta zone appears to be similar between POAG and CACG eyes in advanced glaucoma. Uchida et al. [6] reported that the conventional beta zone area in CACG is much smaller than that in POAG. But one may assume that if the gamma zone area in eyes with minor myopia was deducted from the measured (traditional) beta zone area in those POAG eyes, the difference would be smaller. The similar size and shape of beta zone in both POAG and CACG eyes suggests that the 
Table 3 Parameters show significant association with peripapillary choroidal thickness (PCT) in univariate and multivariate linear regression

\begin{tabular}{|c|c|c|c|c|c|c|}
\hline \multirow[t]{2}{*}{ PCT location } & \multirow[t]{2}{*}{ Mean $\operatorname{PCT}(\mu \mathrm{m})$} & \multicolumn{2}{|c|}{ Univariate analysis } & \multicolumn{2}{|c|}{ Multivariate analysis } & \multirow[t]{2}{*}{$R^{2}$} \\
\hline & & Parameter & $P$-value & Parameter & $P$-value & \\
\hline Temporal inferior & $112.44 \pm 41.75$ & $\begin{array}{l}\text { Age } \\
\text { Beta zone area }\end{array}$ & $\begin{array}{r}<0.001 \\
0.002\end{array}$ & Age & $<0.001$ & 0.220 \\
\hline Temporal & $117.90 \pm 44.31$ & Age & $<0.001$ & Age & $<0.001$ & 0.237 \\
\hline Temporal superior & $127.21 \pm 43.88$ & $\begin{array}{l}\text { Age } \\
\text { Beta zone area }\end{array}$ & $\begin{array}{r}<0.001 \\
0.001\end{array}$ & Age & 0.002 & 0.192 \\
\hline Nasal superior & $120.37 \pm 38.98$ & $\begin{array}{l}\text { Age } \\
\text { Beta zone area }\end{array}$ & $\begin{array}{r}<0.001 \\
0.016\end{array}$ & Age & 0.002 & 0.254 \\
\hline Nasal & $117.13 \pm 41.25$ & Age & 0.003 & Age & 0.003 & 0.106 \\
\hline Nasal inferior & $110.42 \pm 40.77$ & $\begin{array}{l}\text { Age } \\
\text { Beta zone area }\end{array}$ & $\begin{array}{r}<0.001 \\
0.016\end{array}$ & Age & $<0.001$ & 0.229 \\
\hline
\end{tabular}

development of beta zone may share a similar pathophysiology in both glaucoma types.

An interesting finding is that the area of parapapillary gamma zone was negatively associated with the disc ovality index but positively associated with the BMO ovality ratio in our study. The prevalence of gamma zone in non-myopic eyes is much lower than that in myopic eyes [19]. Previous longitudinal studies in children have showed that peripapillary gamma zone may be an acquired feature during axial elongation in myopic eyes [20-22]. However, the mechanism underlying gamma zone development in nonmyopic eyes has remained unclear so far. According to the EDI-OCT images, the parapapillary gamma zone appears to be the reflection of the externally oblique border tissue in non-myopic eyes. In contrary to the predominantly temporal location in myopic eyes, we found that the parapapillary gamma zone was located quite often in peripapillary inferior and nasal regions, suggesting factors other than axial elongation may be involved in the development of gamma zone in non-myopic eyes. Since the ovality of optic disc has long been accepted to be an index of disc tilt [23, 24], the correlation between the gamma zone area and the decreased disc ovality index and increased BMO ovality ratio suggest that parapapillary gamma zone may perhaps play a biomechanical role in disc stability in tilted non-myopic eyes. One could assume that without the presence of gamma zone in tilted optic disc, the shape of BMO would be less round and hence less biomechanically stable. Yamada et al. [25] reported that a larger gamma zone is associated with slow visual field progression in POAG, suggesting a potential protective role of gamma zone. Further studies are needed to investigate the biomechanical roles of border tissue and parapapillary gamma zone in the development of glaucomatous optic neuropathy.

Potential limitations of our study should be mentioned. First, it was a hospital-based research rather than a population-based study, which may contain the possibility of selection bias. Second, our study was a cross-sectional research so that we were not able to perceive the dynamic process of parapapillary atrophy development and its relationship with the susceptibility of glaucomatous optic neuropathy. Third, the study sample size was relatively small, so that clinically significant associations might not have reached statistical significance. Future investigation on the peripapillary border tissues in eyes with tilted optic discs including their biomechanics may further contribute to the understanding of optic nerve head pathophysiology in myopia and glaucoma.

In conclusion, our findings demonstrated that in mainly non-myopic subjects with or without CACG, parapapillary gamma zone was correlated with disc ovality and parapapillary beta zone was correlated with age and presence of glaucoma. The development of beta zone may partially share common mechanism in both POAG and CACG eyes, and not necessarily be associated with parapapillary choroidal vascular defect. The development of gamma zone and its underlying border tissue may play a role in the biomechanical stability of the optic disc.

\section{Summary}

\section{What was known before}

- The OCT defined (new) beta zone is related with POAG, while gamma zone is mainly related with myopia; A close relationship was suggested between peripapillary choroidal thickness and beta zone in POAG

\section{What this study adds}

- In mainly non-myopic subjects with or without CACG, parapapillary gamma zone was correlated with disc ovality and parapapillary (new) beta zone was correlated with age and presence of glaucoma; The development of (new) beta zone may partially share common 
mechanism in both POAG and CACG eyes, and not necessarily be associated with peripapillary choroidal vascular defect.

Acknowledgements This study was supported by a grant from the Science and Technology Commission of the Shanghai Municipality (No.16411962000). The authors thank Prof. Jost Jonas for his critical comments on the manuscript.

\section{Compliance with ethical standards}

Conflict of interest The authors declare that they have no conflict of interest.

Publisher's note: Springer Nature remains neutral with regard to jurisdictional claims in published maps and institutional affiliations.

\section{References}

1. Jonas JB, Nguyen XN, Gusek GC, Naumann GO. Parapapillary chorioretinal atrophy in normal and glaucoma eyes. I. Morphometric data. Invest Ophthalmol Vis Sci. 1989;30:908-18.

2. Jonas JB, Jonas SB, Jonas RA, Holbach L, Dai Y, Sun X, et al. Parapapillary atrophy: histological gamma zone and delta zone. PLoS ONE. 2012;7:e47237.

3. Dai Y, Jonas JB, Huang HL, Wang M, Sun X. Microstructure of parapapillary atrophy: beta zone and gamma zone. Invest Ophthalmol Vis Sci. 2013;54:2013-8.

4. Kim M, Kim T-W, Weinreb RN, Lee EJ. Differentiation of parapapillary atrophy using spectral-domain optical coherence tomography. Ophthalmology. 2013;120:1790-7.

5. Yoo Y, Lee E, Kim T. Intereye difference in the microstructure of parapapillary atrophy in unilateral primary open angle glaucoma. Invest Ophthalmol Vis Sci. 2016;57:4187-93.

6. Uchida H, Yamada T, Tomita G, Kitazawa Y. Peripapillary atrophy in primary angle-closure glaucoma: a comparative study with primary open-angle glaucoma. Am J Ophthalmol. 1999;127:121-8.

7. Foster PJ, Buhrmann R, Quigley HA, Johnson GJ. The definition and classification of glaucoma in prevalence surveys. Br J Ophthalmol. 2002;86:238-42.

8. Dai Y, Jonas JB, Ling Z, Sun X. Ophthalmoscopic-perspectively dis-torted optic disc diameters and real disc diameters. Invest Ophthalmol Vis Sci. 2015;56:7076-83.

9. Sullivan-Mee M, Patel NB, Pensyl D, Qualls C. Relationship between juxtapapillary choroidal volume and beta-zone parapapillary atrophy in eyes with and without primary open-angle glaucoma. Am J Ophthalmol. 2015;160:637-47.

10. Lee SH, Lee EJ, Kim TW. Topographic correlation between juxtapapillary choroidal thickness and microstructure of parapapillary atrophy. Ophthalmology. 2016;123:1965-73.
11. Lee SH, Lee EJ, Kim TW. Topographic correlation between juxtapapillary choroidal thickness and parapapillary deep-layer microvasculature dropout in primary open-angle glaucoma. $\mathrm{Br} \mathbf{J}$ Ophthalmol. 2018;102:1134-40.

12. Flammer J, Orgul S, Costa VP, Orzalesi N, Krieglstein GK, Serra LM, et al. The impact of ocular blood flow in glaucoma. Prog Retin Eye Res. 2002;21:359-93.

13. Galambos P, Vafiadis J, Vilchez SE, Wagenfeld L, Matthiessen ET, Richard G, et al. Compromised autoregulatory control of ocular hemodynamics in glaucoma patients after postural change. Ophthalmology. 2006;113:1832-6.

14. Tobe LA, Harris A, Hussain RM, Eckert G, Huck A, Park J, et al. The role of retrobulbar and retinal circulation on optic nerve head and retinal nerve fiber layer structure in patients with open-angle glaucoma over an 18-month period. $\mathrm{Br} \mathrm{J}$ Ophthalmol. 2015;99:609-12.

15. Onda E, Cioffi GA, Bacon DR, Van Buskirk EM. Microvasculature of the human optic nerve. Am J Ophthalmol. 1995;120:92-102.

16. Quigley HA, Katz J, Derick RJ, Gilbert D, Sommer A. An evaluation of optic disc and nerve fiber layer examinations in monitoring progression of early glaucoma damage. Ophthalmology. 1992;99:19-28.

17. Jonas JB, Martus P, Budde WM, Jünemann A, Hayler J. Small neuroretinal rim and large parapapillary atrophy as predictive factors for progression of glaucomatous optic neuropathy. Ophthalmology. 2002;109:1561-7.

18. Quigley HA, Enger C, Katz J, Sommer A, Scott R, Gilbert D. Risk factors for the development of glaucomatous visual field loss in ocular hypertension. Arch Ophthalmol. 1994;112:644-9.

19. Zhang Q, Wang YX, Wei WB, Xu L, Jonas JB. Parapapillary Beta Zone and Gamma Zone in a Healthy Population: The Beijing Eye Study 2011. Invest Ophthalmol Vis Sci. 2018;59:3320-9.

20. Kim M, Choung HK, Lee KM, Oh S, Kim SH. Longitudinal Changes of Optic Nerve Head and Peripapillary Structure during Childhood Myopia Progression on OCT: Boramae Myopia Cohort Study Report 1. Ophthalmology. 2018;125:1215-23.

21. Lee KM, Choung HK, Kim M, Oh S, Kim SH. Positional Change of Optic Nerve Head Vasculature during Axial Elongation as Evidence of Lamina Cribrosa Shifting: Boramae Myopia Cohort Study Report 2. Ophthalmology. 2018;125:1224-33.

22. Lee KM, Choung HK, Kim M, Oh S, Kim SH. Change of betaZone Parapapillary Atrophy During Axial Elongation: Boramae Myopia Cohort Study Report 3. Invest Ophthalmol Vis Sci. 2018;59:4020-30.

23. You QS, Xu L, Jonas JB. Tilted optic discs: The Beijing Eye Study. Eye. 2008;22:728-9.

24. Tay E, Seah SK, Chan SP, Lim AT, Chew SJ, Foster PJ, et al. Optic disk ovality as an index of tilt and its relationship to myopia and perimetry. Am J Ophthalmol. 2005;139:247-52.

25. Yamada H, Akagi T, Nakanishi H, Ikeda HO, Kimura Y, Suda K, et al. Microstructure of peripapillary atrophy and subsequent visual field progression in treated primary open-angle glaucoma. Ophthalmology. 2016;123:542-51. 